Joan Sebastián Soto Triana*

Catalina Gómez Villamizar ${ }^{\star \star \star}$ 


\section{Avances en realidad virtual e intervenciones en psicología clinica*}

Cómo citar este artículo: Soto Triana, J. S., \& Gómez Villamizar, C. (2018). Avances en realidad virtual e intervenciones en psicología clínica. Tesis Psicológica, 13(1), 68-81. https://doi.org/10.37511/tesis.v13n1a5
Recibido: 22 enero 2018

Revisado: 31 enero 2018

Aprobado: 25 junio 2018

\section{ABSTRACT}

This paper presents an overview of the advances in the use of virtual reality (VR) as a technological tool incorporated into the research and intervention in clinical psychology. The contributions of VR in the clinical intervention of anxiety and eating disorders are characterized, as well as the possibilities it offers for the investigation of phenomena such as psychosis or the configuration of complex systems that allow different therapeutic functions such as the EMMA system. Finally, the usefulness and possibilities offered by VR systems are discussed.

Keywords: virtual reality, clinical psychology, psychological treatments, exposure therapy, anxiety disorders, eating disorders.

\section{RESUMEN}

El artículo presenta un panorama general de los avances en la utilización de realidad virtual (RV) como herramienta tecnológica incorporada en la actividad investigativa e interventiva en psicología clínica. Se encuentran caracterizados los aportes de la RV en la intervención clínica de trastornos de ansiedad y alimentarios, así como las posibilidades que brinda para la investigación de fenómenos como la psicosis o la configuración de sistemas complejos que permiten diferentes funciones terapéuticas como el sistema EMMA. Al final se plantea una discusión sobre la utilidad y las posibilidades que brindan los sistemas de RV en la actualidad.

Palabras clave: realidad virtual, psicología clínica, tratamientos psicológicos, terapia de exposición, trastornos de ansiedad, trastornos alimentarios. 


\section{Introducción}

La realidad virtual (RV) es un desarrollo tecnológico que ha venido tomando fuerza en los últimos años, su progreso e implementación han tenido diversos nichos y se ha utilizado principalmente en el desarrollo de ambientes para producir simulaciones de aspectos formales de la cotidianidad que pueden ser controlados y evaluados. La RV permite generar ambientes tridimensionales a través de un computador y da la posibilidad a un usuario de interactuar con los diferentes elementos ambientales de la realidad programada, lo que permitela formalización del control de variables y la posibilidad de registro que en la realidad material resultaría dispendioso (Zimand et al., 2003; Botella et al., 2004; Bretón-López et al., 2017).

Pp. 68 - 81

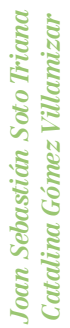

Esta característica de la generación de ambientes que en principio fue explotada por las empresas desarrolladoras de software para simulación de juegos, ahora se torna un valor importante cuando se piensa en la expansión hacia otros usos, por ejemplo, terapéutico. Una de las principales características que deriva en la utilización de la tecnología de RV en disciplinas como la psicología es la posibilidad de poner a un sujeto en un ambiente específico, con condiciones manipulables y que este no tenga sensación de peligro sobre su integridad (Botella et al. 2009; Miró, 2007; Gutiérrez-Maldonado et al., 2007).

La utilización de la tecnología de RV en torno a procedimientos clínicos en psicología ha sido ampliamente desarrollada, como lo indica Botella et al. (2006) la potencialidad de la herramienta permite generar un espacio terapéutico que ayuda a controlar las fuentes de estimulación y la posibilidad de control por parte del terapeuta sobre la medida de la exposición. En este sentido, la terapia por exposición, que es la base de la administración de la RV, se fortalece en tanto la experiencia vivida por el paciente puede ser activa manteniendo la seguridad que brinda la noción de un ambiente virtual controlado (Price \& Anderson, 2007; Meyerbröker \& Emmelkamp, 2010).

El estudio de García-Palacios et al. (2007) mostró que la terapia de exposición aplicada para el tratamiento de fobias específicas a través de RV tiene una aceptación del $76.6 \%$ en contraposición a la habitual terapia de exposición en vivo. El estudio sugiere que la terapia a través de RV logra una sensación de seguridad que ayuda a la preparación del paciente y reduce los índices de ansiedad para la aplicación de la estimulación, lo que se confirma también en estudios posteriores (eg. Motraghi et al. 2014; Botella et al., 2015). Lo anterior demuestra las implicaciones que tiene la RV en el desarrollo de terapias de exposición, en tanto se muestra como una opción valorada como segura por los pacientes, en tanto que no hay un contacto directo con estímulos aversivos (Bush, 2008).

En este trabajo se plantea una revisión de los usos de la RV en campos específicos de aplicación desde hace una década, con el objetivo de hacer seguimiento a las líneas definidas en el proceso de desarrollo de los primeros trabajos sobre el tema, así como la ampliación a otros sectores aplicados.

\section{Realidad virtual, ansiedad y fobias específicas}

En primera instancia se asume que una fobia es un aprendizaje que se da por principios de condicionamiento aversivo (Domjan, 2007) e implica la asociación entre estímulos ambientales, respuestas emocionales y comportamentales que se agrupan en lo que la psicología clínica ha definido como ansiedad (Bańos et al., 2008). Adicionalmente, se ha demostrado que a estos efectos emocionales y comportamentales se han asociado pensamientos y significaciones que 
permiten nombrar, relatar y explicar las percepciones y sentimientos que se producen por la exposición a estímulos ambientales aversivos (García-García et al., 2011; Baños et al., 2013).

En la revisión de la literatura, Botella et al. (2007) informaron que el área mayormente explorada, que generó uno de los espacios de trabajo y desarrollo sobre los usos de realidad virtual en intervención clínica, es el tratamiento de fobias. La categoría que usaron Botella et al. (2007) para mostrar la diversidad de estudios sobre el tema fue la de "Realidad virtual y trastornos de ansiedad". Bajo esta categoría caracterizaron las principales fobias en las que se realizaron desarrollos interventivos: fobia social, agorafobia o trastorno de pánico, acrofobia o temor a las alturas, claustrofobia, aerofobia y aracnofobia. Estos hallazgos son respaldados por Opris et al. (2012) quienes caracterizan los campos donde se insiste en la evaluación de tratamientos terapéuticos que incluyen la RV como estrategia de exposición: miedo a volar, trastorno de pánico / agorafobia, fobia social, aracnofobia y acrofobia.

En su estudio, además de caracterizar los campos en los que se ha desarrollado la RV como medio tecnológico para la terapia de exposición, Opris et al. (2012) realizan una medición de la eficacia de las intervenciones, en sus resultados muestran un nivel significativamente alto de efectividad en el tratamiento de las fobias a través de la terapia de exposición utilizando la $\mathrm{RV}$ : $(\mathrm{D}=1.12$; VAR $\mathrm{D}=.34,95 \%$ CI[0.71- 1.52], Po.05) (Opris et al., 2012, p. 89). Ahora bien, a partir de la comparación de 15 estudios que utilizaron exposición con RV, no se evidencian resultados diferentes a los obtenidos con los métodos clásicos de exposición para las fobias $(\mathrm{D}=.16, \mathrm{VAR}=5.16$, 95\% CI [-0.03-0.36], P>.05).

Morina et al. (2015), en un análisis sobre la efectividad del tratamiento de exposición a través de RV sobre fobias específicas, muestran la eficacia que tiene la intervención en sus efectos posteriores en la vida real. La revisión de los autores incluyó 14 ensayos clínicos sobre fobias específicas, encontraron que los pacientes sometidos a RV obtuvieron mejores resultados en evaluaciones conductuales en el postratamiento que pacientes en lista de espera - grupo control- $(\mathrm{g}=1.41)$. Sumado a esto, se encontró que no existen diferencias significativas entre la exposición por $\mathrm{RV}$ y la exposición en vivo ( $\mathrm{g}=$ -0.09 y 0.53 ) (Morina et al., 2015, p. 22).

Se ha observado evidencia que muestra la efectividad de los procedimientos de exposición a través de RV en diferentes trastornos de ansiedad que incluyen fobias específicas, se ha mostrado la importancia de la intervención en función de los resultados obtenidos y de la posibilidad de adherencia al tratamiento, así como la oportunidad para que los pacientes sientan seguridad para la exposición. Adicionalmente, se observa que la intervención clínica no tiene diferencias significativas con respecto a la exposición en vivo, lo que supone la efectividad del recurso con una mayor posibilidad de seguridad para el paciente.

\section{Realidad virtual y trastornos alimentarios}

Siguiendo las categorías analíticas de Gutiérrez (2002) y Botella et al. (2007) para la aplicación de la RV en intervenciones clínicas desde la psicología, se realiza una revisión de las intervenciones sobre trastornos alimentarios. Gutiérrez-Maldonado et al. (2010) desarrollaron 4 ambientes de RV para la evaluación de trastornos alimentarios y su relación con la percepción de distorsión de la imagen corporal así como la insatisfacción con el cuerpo. Utilizando una muestra experimental de 85 personas con desorden alimenticio, divididos en 3 grupos de personas diagnosticadas con anorexia

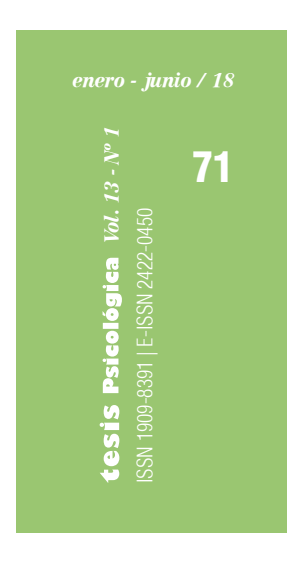


nerviosa, bulimia y trastornos alimenticios no especificados y un grupo control de 108 personas, se procedió a evaluar las respuestas sobre la percepción de la imagen corporal y la insatisfacción en el momento de interactuar a través de los ambientes de RV con diferentes situaciones.

Gutiérrez-Maldonado et al. (2010) lograron generar cuatro ambientes donde se presentan diferentes situaciones de la vida cotidiana: en el primero los participantes se encuentran en una cocina y se pueden sentar en una mesa con alimentos ligeros en calorías, en el segundo ambiente los participantes se ubican sentados en una cocina con alimentos con un contenido calórico alto. En los dos primeros ambientes los participantes se encuentran solos en el escenario. El tercer escenario se desarrolla en un ambiente de restaurante, el participante se ubica en una mesa y encuentra comida ligera en calorías. En el cuarto ambiente el participante se encuentra en un restaurante, se ubica en una mesa y encuentra comida con alto contenido calórico. En los ambientes de restaurante las personas se encuentran acompañadas por personajes virtuales sentados en la misma mesa.

El anterior estudio muestra las posibilidades que se abren para la exploración de los fenómenos asociados a los trastornos alimenticios, en este caso los participantes son expuestos a situaciones de la vida cotidiana en un ambiente virtual que permite realizar mediciones sobre sus respuestas comportamentales y sobre las apreciaciones sobre sí mismos en el momento de interactuar con alimentos en contextos que pueden incluir o no la presencia de otras personas (Gutiérrez-Maldonado et al., 2010). El estudio permitió observar una relación importante entre los participantes que han sido diagnosticados previamente con algún desorden alimenticio y su percepción distorsionada de la imagen corporal con las condiciones en las que se encuentra en términos contextuales, cuando la comida tiene mayor cantidad de calorías se percibe mayor distorsión. Sin embargo, no existe una relación significativa que indique el aumento de la distorsión de la imagen corporal en presencia de otras personas en el contexto (Gutiérrez-Maldonado et al., 2010).

Este tipo de estudios permiten observar el uso de la tecnología de RV no solo para la intervención, sino también como herramienta de investigación que permite observar condiciones particulares de los fenómenos a estudiar. El uso de RV se ha probado sistemáticamente para la intervención clínica a personas con desórdenes alimenticios que implican una distorsión e insatisfacción de la imagen corporal. Marco et al. (2013) utilizaron 5 ambientes de RV para aplicar un protocolo de terapia cognitivo conductual que incluye cinco áreas de trabajo, en cada una de estas áreas el ambiente permite al participante hacer una evaluación de su imagen corporal, observando escalas de su concepción corporal que se deben comparar con las escalas reales de su cuerpo, lo que permite una reflexión sobre la imagen y la posibilidad de observar la distorsión que se tiene y las razones de ella.

Una posibilidad adicional para la utilización de la RV la exponen Gorini et al. (2010), quienes desarrollaron un trabajo sobre la emocionalidad producida por la interacción con distintos alimentos en personas diagnosticadas con desórdenes de la alimentación. El trabajo utilizó tres condiciones de RV donde el participante podía interactuar con alimentos en un espacio virtual, la característica principal de los alimentos es que suponían una gran carga calórica y en cada condición cambiaba el tiempo de exposición de los alimentos. Las mediciones muestran un aumento significativo en los niveles de ansiedad de las personas con desórdenes alimenticios en presencia de los alimentos con alto contenido calórico. 
Se observa a través de los estudios presentados que la RV se ofrece como una posibilidad que no se limita a las posibilidades de intervención clínica, sino que es utilizada en diferentes formas para ahondar en aspectos específicos de los fenómenos que integran la emocionalidad, la creencia y el comportamiento en torno a los trastornos alimenticios. Así mismo, se ofrece como una herramienta útil para acercar los contextos complejos a escenarios controlados donde las variables pueden resultar de mayor manejo para probar diversas configuraciones que están en la base de la explicación de los fenómenos y su posible intervención.

\section{Realidad virtual y psicosis}

Daniel Freeman (2008) refiere la RV como una herramienta que permite abrir el campo de trabajo sobre campos de la psicopatología y la intervención clínica, afirma que la posibilidad que trae la RV en términos de la identificación y control de variables asociadas a diversos fenómenos psicopatológicos permite al psicólogo clínico una posibilidad investigativa única en ambientes preparados según condiciones específicas que replican los contextos cotidianos con la bondad del control de factores para generar explicaciones correlacionales o causales.

Uno de los posibles usos que permite esta herramienta es la posibilidad de utilizar ambientes sociales creados para explorar la manera en que sujetos experimentan los síntomas de ciertas patologías mentales como la ansiedad social o los trastornos adaptativos (Freeman, 2008; Rivera et al., 2008; Quero et al., 2017). Estos ambientes sociales recreados pueden ser transferidos a otras experiencias clínicas que permiten identificar variables asociadas a la presencia de síntomas en personas diagnosticadas con trastornos psicóticos (Freeman, 2008; Slater, 2009; Hesse et al., 2017; Counotte et al., 2017).
Freeman (2008) expone al menos 7 ventajas que trae la utilización de RV: 1. Evaluación de síntomas, en este caso la puesta en situación de los sujetos permite observar las reacciones desfavorables y llevar un registro de los comportamientos, que permite complementar los resultados de inventarios de evaluación y procesos de entrevista en casos donde se supone ocurren ilusiones persecutorias y paranoia. 2 . Establecer correlaciones entre síntomas y respuestas fisiológicas y comportamientos; en este caso la posibilidad de un ambiente virtual permite realizar mediciones en tiempo real de las condiciones fisiológicas de activación asociadas a la producción de comportamientos específicos que permite vincular aspectos causales entre la aparición de síntomas, los estímulos ambientales y las respuestas fisiológicas y comportamentales. 3. Identificación de variables predictoras: permite la identificación de variables individuales sobre aspectos específicos de los síntomas de preocupación, ilusión persecutoria, paranoia, etc. 4. Identificación de predictores diferenciales, la RV permite generar estudios correlacionales que vinculan variables asociadas a diferentes desórdenes clínicos que pueden estar presentes en diferentes trastornos como la ansiedad social y trastornos adaptativos; sin embargo, se pueden realizar diferencias específicas entre las respuestas en uno y otro caso vinculadas situaciones como estímulos antecedentes sobre los cuales se pueden generar predicciones de respuestas específicas en cada caso diferenciado. Para el caso de la psicosis se pueden vincular situaciones de ansiedad que llevan a respuestas específicas que permiten generar marcadores de predicción sobre los comportamientos esperables. 5. Identificar predictores ambientales: siguiendo lo descrito en el anterior aparte no solo se pueden establecer marcadores de predicción sobre respuestas diferenciales entre distintos desórdenes, sino también establecer relaciones entre estímulos específicos del ambiente y las

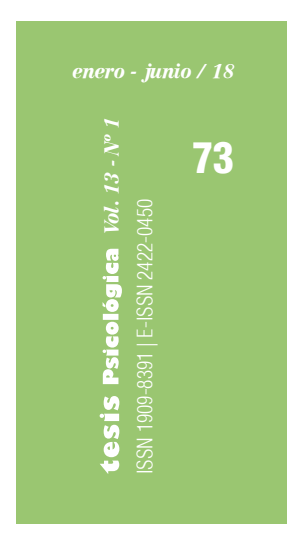


afines a las preferencias de los niños. Los ambientes se modificaron para que las gráficas tuviesen una tendencia animada y El libro de la vida se modificó para que tuviese una presentación gráfica tecnológica contemporánea. Los resultados de la intervención muestran una significativa mejoría en el control de la ansiedad producto del maltrato y la posibilidad de un manejo adecuado de las respuestas emocionales, que permite decir que el mundo de EMMAinfancia se torna una herramienta que permite no solo la intervención clínica en adultos, sino que se ha extendido a otro tipo de poblaciones en función de su edad (López-Soler et al., 2011; Alcántara et al., 2017).

Por otro lado, EMMA se ha utilizado también en la intervención de trastornos adaptativos en vista de su posibilidad para adaptar los recursos ambientales a la posibilidad de generar procesos emocionales que son desplegados a través de la estimulación que utiliza el terapeuta (AndreuMateu et al., 2012). Se utiliza también para la resignificación de contenidos emocionales en la elaboración de duelos y se torna en una herramienta eficaz para este objetivo el diario de vida (Botella et al., 2008).

\section{Discusión}

Como se aprecia en los apartes anteriores, la RV ha reclamado un espacio amplio dentro de la intervención clínica. Las características más importantes para su posicionamiento son las que tienen que ver con la configuración de entornos virtuales donde se puede emular la realidad, la concreción de un contexto controlado de ambientes que reproduce la realidad de los individuos. Sumado a esto, se encuentra la posibilidad de control sobre los grados de exposición estimulante que directamente afectan las respuestas del sujeto a intervenir, esta misma característica deriva en formas de control que ayudan al estudio y a la aplicación interventiva sobre problemas clínicos específicos (Freeman, 2008).

Como se ha mostrado, la terapia a través de RV permite la consolidación de un proceso de adherencia al tratamiento en función de las formas de exposición que son percibidas por el individuo como contextos controlados, donde la exposición está totalmente vigilada por el clínico y la idea de que sea a través de un sistema tecnológico programado permiten mantener una sensación de seguridad que no se encuentra en las exposiciones en vivo (García-Palacios et al. 2007; Motraghi et al 2014; Botella et al., 2015).

Esto último es significativo y se vuelve una opción muy importante para la labor de los psicólogos clínicos, en tanto los escenarios pueden responder a las demandas de los individuos que Pp. $68-81$ requieren de contextos estimulantes específicos. Ejemplos de esta posibilidad son los sistemas que integran recursos diversos como EMMA. Estos aplicativos que poseen grandes capacidades para la utilización de estímulos o situaciones simuladas permiten al clínico adaptar rápidamente formas de intervención que se adecuan a los intereses del consultante (Botella et al., 2007, 2009).

La elaboración de contextos específicos de RV permite observar la manera en que las personas interactúan con los estímulos ambientales y producen sistemas organizados de creencias sobre la relación contextual y su sí mismo, tal es el caso de los trastornos alimentarios, que han mostrado la manera en que se consolidan interpretaciones subjetivas de acuerdo a la información estimulante presente en el ambiente (Gutiérrez- Maldonado et al., 2010). Esta característica ubica la RV como una herramienta de investigación que permite controlar la exposición del individuo a un ambiente preparado para realizar mediciones sobre su comportamiento y las configuraciones cognitivas que surgen de la

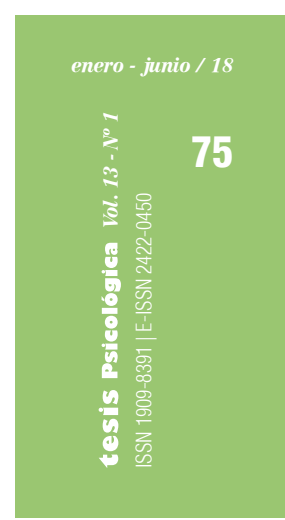


interacción con ciertos estímulos. Un ejemplo adicional es la manera en que la RV ha colaborado en la investigación sobre la psicosis, que permite evaluar las condiciones en las que la persona se comporta en función de la presencia de ciertos estímulos sociales (Freeman, 2008).

Ahora bien, no solo la RV permite la acomodación en función de las demandas clínicas específicas de los consultantes, sino que también se puede adecuar a problemáticas históricosociales en las que viven los sujetos. De la Rosa Gómez y Cárdenas-López (2012) presentan un diseño de RV que permite la intervención clínica en víctimas de violencia criminal, que responde a las condiciones contextuales por las que atraviesan las comunidades mexicanas, a causa de la violencia asociada con el tráfico de drogas.

Pp. $68-81$

Los autores también refieren la funcionalidad clínica sobre la exposición en RV, que muestra mejores resultados en el control de los síntomas en contra posición a la terapia por imaginación; además, detallaron que los participantes encontraron menos aversivos los escenarios de RV (de la Rosa Gómez y Cárdenas- López, 2012). Kramer et al. (2013) confirman que los escenarios de $\mathrm{RV}$ ayudan a poner en situación y permiten una inmersión contextual significativa en entornos específicos, por ejemplo, en poblaciones de excombatientes. En su estudio, Kramer et al. (2013) indagaron la percepción de militares veteranos hacia la $\mathrm{RV}$ como herramienta de evaluación y tratamiento de TEPT. A través de entrevistas semiestructuradas pudieron caracterizar que los veteranos sintieron que la herramienta permite una buena inmersión en los entornos de combate, ya que hay una percepción subjetiva de reactividad fisiológica, pensamientos y comportamientos similares a los experimentados que desencadenaron recuerdos.

De otro lado, Kramer et al. (2013) presentan una percepción asociada a la experiencia de inmersión en ambientes de RV. Los veteranos informan que es preocupante la manera en que los contextos recreados evocan recuerdos traumáticos fuertes e impactan de manera negativa la emocionalidad. Esta preocupación es recurrente en los argumentos sobre las terapias de exposición, específicamente las que refieren tratamientos sobre el TEPT consolidado en procesos de violencia criminal o producto de conflictos sociales. La reiterada mención a la revictimización es un problema que tiene que tratar la terapia de exposición y evidentemente la RV ha sido blanco de los argumentos en contra del uso de tecnologías de información para reproducir escenarios de violencia.

De acuerdo a lo anterior, el desarrollo de la RV debe contemplar estudios que permitan identificar si se dan y en qué medida procesos de revictimización y si esta condición es necesaria para la elaboración del trauma. De acuerdo a las clásicas formas explicativas de la terapia de exposición, la recurrencia sobre los escenarios estimulares es necesaria para producir el contra condicionamiento y para lograr el control de los efectos ansiosos (McLean \& Foa, 2013). Soto et al. (2017) muestran la utilidad de configurar escenarios de RV que permiten situar a los individuos en situaciones específicas de combate, utilizando contextos con estímulos generales de las situaciones como factores ambientales, sonidos de la fauna y flora, disparos, etc., que permiten generar la reacción de los síntomas ansiosos sin evocar los aspectos específicos de la consolidación del trauma. La posibilidad de desarrollar herramientas virtuales puede favorecer el tratamiento de personas que han sido expuestas a situaciones de combate en un país como Colombia que ha tenido diversos escenarios de conflicto armado. Teniendo en cuenta la necesidad de desarrollar propuestas que no involucren escenarios de revictimización, la RV ofrece una importante herramienta de investigación e intervención. Al seguir los desarrollos 
presentados se pueden lograr avances importantes para configurar ambientes de realidad virtual que tengan en cuenta los aspectos estimulantes específicos en la consolidación de los procesos ansiosos en personas que han sido actores o testigos del conflicto en el país.

\section{Conclusiones}

La RV se configura como una herramienta tecnológica que ha mostrado resultados importantes en el campo clínico, específicamente en la intervención de trastornos de ansiedad. Los resultados muestran una amplia favorabilidad en la percepción de los consultantes acerca de la seguridad y el control de la exposición a estímulos aversivos a través de medios virtuales, lo que implica una adherencia al tratamiento por exposición de acuerdo a la sensación de seguridad que trasmite la herramienta.

Se ha probado la efectividad de las intervenciones a través de RV, de acuerdo a mediciones que comparan la efectividad con exposiciones en vivo. Los estudios muestran que los resultados de consultantes que han sido intervenidos con terapias de exposición en vivo y con RV se mantienen en el tiempo, lo que implica que la herramienta no influye negativamente y sí permite la percepción de seguridad y control ante los estímulos aversivos.

La psicología clínica ha ampliado los usos de la RV para diferentes trastornos como los alimentarios y psicóticos. Esta apertura ha logrado consolidar estudios que exploran las bases mismas de la producción de los fenómenos psicológicos asociados para ampliar el entendimiento sobre los mismos. Se mostró como los ambientes de RV desarrollados permiten ampliar las expectativas de uso, que llegan incluso a la producción de sistemas de ambientes de RV como EMMA que proveen diferentes contextos para el diagnóstico e intervención de diferentes problemáticas en un mismo paquete. 


\section{Referencias}

Andreu-Mateu, S., Botella, C., Quero, S., Guillén, V., \& Baños, R.M. (2012). La utilización de la realidad virtual y estrategias de psicología positiva en el tratamiento de los trastornos adaptativos. Behavioral Psychology/Psicologia Conductual, 20(2) 323-348.

Alcántara, M., Castro, M., Martínez, A., Fernández, V., \& López-Soler, C. (2017). El sistema de realidad virtual EMMA-Child para el tratamiento del trauma infantil: experiencias iniciales. Revista de Psicología Clínica con Niños y Adolescentes, 4(3), 26-34.

Baños, R.M., Botella, C., García-Palacios, A., Quero, S., Alcañiz, M., \& Guillen, V. (2008). Behandlung psychischer Probleme im virtuellen Raum. En S. Bauer, \& H. Kordy (Eds.) EMental-Health: Neue Medien in der psychosozilen Versorgung. Heidelberg: Springer-Verlag.

Baños, R. M., Botella, C., Guillen, V., García-Palacios, A., Quero, S., Bretón-López, J., \& Alcañiz, M. (2009). An adaptive display to treat stress-related disorders: EMMA's World. British Journal of Guidance \& Counselling, 37, 347-356.

Baños, R. M., Guillén, V., García-Palacios, A., Quero, S., \& Botella, C. (2013). Las nuevas tecnologías en el tratamiento de los trastornos de ansiedad. Información Psicológica, (102), $28-46$.

Bretón-López, J., \& Mira, A., Castilla, D., García-Palacios, A., \& Botella, C. (2017). Revisión de aplicaciones de las tecnologías de la información y la comunicación en psicología clínica y de la salud en infancia y adolescencia. Revista de Psicología Clínica con Niños y Adolescentes, 4(3), 11-16.

Botella, C., Quero, S., Baños, R. M., Perpiñá, C., García-Palacios, A., \& Riva, G. (2004). Virtual reality and psychotherapy. Cybertherapy, 99, 37-52.

Botella, C., García-Palacios, A., Baños, R. M. (2005). The treatment of emotions in a virtual world. Application in a case of posttraumatic stress disorder. En Cyber Therapy Conference 2005, Basilea (Suiza).

Botella, C., García-Palacios, A., Quero, S., Baños, R. M., \& Bretón-López, J. M. (2006). Título: Realidad Virtual y tratamientos psicológicos: Una revisión. Psicología Conductual, 3, 491-510.

Botella, C., Baños, R., García-Palacios, A., Quero, S., Guillén, V., \& José Marco, H. (2007). La utilización de las nuevas tecnologías de la información y la comunicación en psicología clínica. UOC Papers. Revista sobre la Sociedad del Conocimiento, (4), 32-41. 
Botella, C., Osma, J., García Palacios, A., Guillén,V., \& Baños, R. (2008). Treatment of complicated grief using virtual reality. A case report. Death Studies, 32(7), 674-692.

Botella, C., Quero, S., Serrano, B., Baños, R. M., \& García-Palacios, A. (2009). Avances en los tratamientos psicológicos: la utilización de las nuevas tecnologías de la información y la comunicación. Anuario de Psicología, 40(2), 155-170.

Botella, C., Serrano, B., Baños, R. M., \& García-Palacios, A. (2015). Virtual reality exposure-based therapy for the treatment of post-traumatic stress disorder: a review of its efficacy, the adequacy of the treatment protocol, and its acceptability. Neuropsychiatric Disease and Treatment, 11, 2533-2545. Doi: 10.2147/NDT.S89542

Bush, J. (2008). Viability of virtual reality exposure therapy as a treatment alternative. Computers in Human Behavior, 24(3), 1032-1040.

Counotte, J., Pot-Kolder, R., van Roon, A. M., Hoskam, O., van der Gaag, M., \& Veling, W. (2017). High psychosis liability is associated with altered autonomic balance during exposure to Virtual Reality social stressors. Schizophrenia Research, 184, 14-20.

Domjan, M. (2007). Principios de aprendizaje y conducta. Madrid: Editorial Paraninfo.

Freeman, D. (2008). Studying and treating schizophrenia using virtual reality: a new paradigm. Schizophrenia Bulletin, 34(4), 605-610.

García-García, E. S., Rosa-Alcázar, A. I., \& Olivares-Olivares, P. J. (2011). Terapia de exposición mediante realidad virtual e internet en el trastorno de ansiedad/fobia social: Una revisión cualitativa. Terapia Psicológica, 29(2), 233-243.

García-Palacios, A., Botella, C., Hoffman, H., \& Fabregat, S. (2007). Comparing Acceptance and Refusal Rates of Virtual Reality Exposure vs. In Vivo Exposure by Patients with Specific Phobias. Cyberpsychology \& Behavior, 10(5), 722-724. doi:10.1089/cpb.2007.9962

Gorini, A., Griez, E., Petrova, A., \& Riva, G. (2010). Assessment of the emotional responses produced by exposure to real food, virtual food and photographs of food in patients affected by eating disorders. Annals of General Psychiatry, 9(1), 30.

Gutiérrez, J. (2002). Aplicaciones de la Realidad Virtual en psicología Clínica. Aula Médica Psiquiátrica, 4(2), 92-126.

Gutiérrez-Maldonado, J., Alsina-Jurnet, I., Carvallo-Becíu, C., Letosa-Porta, A., \& Magallón-Neri, E. (2007). Aplicaciones clínicas de la realidad virtual en el ámbito escolar. Cuadernos de Medicina Psicosomática y Psiquiatría de Enlace, 82, 32-51. 
Gutiérrez-Maldonado, J., Ferrer-García, M., Caqueo-Urízar, A., \& Moreno, E. (2010). Body image in eating disorders: The influence of exposure to virtual-reality environments. Cyberpsychology, Behavior and Social Networking, 13(5), 521-531.

Hesse, K., Schroeder, P. A., Scheeff, J., Klingberg, S., \& Plewnia, C. (2017). Experimental variation of social stress in virtual reality-Feasibility and first results in patients with psychotic disorders. Journal of Behavior Therapy and Experimental Psychiatry, 56, 129-136.

Kramer, T., Savary , P., Pyne, J., Kimbrell, T., \& Jegley, S. (2013). Veteran perceptions of virtual reality to assess and treat posttraumatic stress disorder. Cyberpsychology, Behavior and Social Networking, 16(4), 293-301.

McLean , C., \& Foa, E. (2013). Dissemination and implmentation of prolonged exposure therapy for posttraumatic stress disorder. Journal of anxiety Disorders, 27(8), 788-792.

Marco, J. H., Perpina, C., \& Botella, C. (2013). Effectiveness of cognitive behavioral therapy supported by virtual reality in the treatment of body image in eating disorders: One year follow-up. Psychiatry Research, 209(3), 619-625.

Meyerbröker, K., \& Emmelkamp, P. M. (2010). Virtual reality exposure therapy in anxiety disorders: a systematic review of process and outcome studies. Depression and Anxiety, 27(10), 933-944.

Miró, J. (2007). Psicoterapia y nuevas tecnologías. Cuadernos de Medicina Psicosomática y Psiquiatría de Enlace, 81, 15-20.

Morina, N., Ijntema, H., Meyerbröker, K., \& Emmelkamp, P. M. (2015). Can virtual reality exposure therapy gains be generalized to real-life? A meta-analysis of studies applying behavioral assessments. Behaviour Research and Therapy, 74, 18-24.

Motraghi, T. E., Seim, R. W., Meyer, E. C., \& Morissette, S. B. (2014). Virtual reality exposure therapy for the treatment of posttraumatic stress disorder: A methodological review using CONSOR. International Journal of Clinical Psychology, 70, 197-208. Doi: 10.4088/JCP.v68n1102

López-Soler, C., Castro, M., Alcántara, M., \& Botella, C. (2011). Sistema de realidad virtual EMMA-Infancia en el tratamiento psicológico de un menor con estrés postraumático. Revista de Psicopatología y Psicología Clínica, 16(3), 189-206. 
Opriş, D., Pintea, S., García-Palacios, A., Botella, C., Szamosközi, Ş., \& David, D. (2012). Virtual reality exposure therapy in anxiety disorders: a quantitative meta-analysis. Depression and Anxiety, 29(2), 85-93.

Price, M., \& Anderson, P. (2007). The role of presence in virtual reality exposure therapy. Journal of Anxiety Disorders, 21(5), 742-751.

Quero Castellano, S., Andreu Mateu, M. S., Moragrega Vergara, I., Baños Rivera, R. M., Molés Amposta, M., Nebot, S., \& Botella, C. (2017). Un programa cognitivo-conductual que utiliza la realidad virtual para el tratamiento de los trastornos adaptativos: una serie de casos. Revista Argentina de Clínica Psicológica, 16(1), 5-18.

Rivera, R. M. B., Botella, V. G., Arbona, C. B., Palacios, A. G., Rodero, M. J., \& Castellano, S. Q. (2008). Un programa de tratamiento para los trastornos adaptativos. Un estudio de caso. Apuntes de Psicología, 26(2), 303-316.

De la Rosa Gómez, A., \& Cárdenas-López, G. (2012). Trastorno por estrés postraumático: eficacia de un programa de tratamiento mediante realidad virtual para víctimas de violencia criminal en población mexicana. Anuario de Psicología, 42(3), 377-391.

Slater, M. (2009). Place illusion and plausibility can lead to realistic behaviour in immersive virtual environments. Philosophical Transactions of the Royal Society of London B: Biological Sciences, 364(1535), 3549-3557.

Soto, J.S., Cárdenas-López, G., Oliveros, E., \& Rojas, C. (2017). Diseño de ambientes de realidad virtual para el tratamiento de trastorno de estrés postraumático en víctimas y testigos de violencia en el conflicto colombiano. En Memorias Congreso Colombiano de Psicología y Construcción de Paz (pp. 1141-1143).

Zimand, E., Rothbaum, B., Tannenbaum, L., Ferrer, M. S., \& Hodges, L. (2003). Technology meets psychology: Integrating virtual reality into clinical practice. The Clinical Psychologist, 56, 5-11. 